\title{
In vitro 3-D multicellular models for cytotoxicity assay and drug screening
}

Ru Zang ${ }^{\# a}$, Xudong Zhang ${ }^{\# b}$, Jianxin Sun ${ }^{c}$, Shang-Tian Yang*

William G. Lowrie Department of Chemical and Biomolecular Engineering, The Ohio State University, $151 \mathrm{~W}$. Woodruff Avenue, Columbus, Ohio 43210, USA

*Corresponding author. Phone: (614) 292-6611; fax: (614) 292-3769; email: yang.15@ osu.edu.

${ }^{\#}$ These two authors made equal contributions.

a Present address: Genzyme, A Sanofi Company, 31 New York Ave., Framingham, MA 01702

${ }^{\mathrm{b}}$ Present address: Shire HGT, 200 Shire Way, Lexington, MA 02421

${ }^{\mathrm{c}}$ Present address: Boehringer Ingelheim, 6701 Kaiser Dr., Fremont, CA 94555

Submitted to Process Biochemistry

(C) 2016. This manuscript version is made available under the Elsevier user license http://www.elsevier.com/open-access/userlicense/1.0/ 


\begin{abstract}
Three-dimensional (3-D) cell culture models have been developed to improve drug screening and predictive efficacy. In this study, a high-throughput drug screening system with autofluorescent cells cultured in 3-D polyethylene terephthalate (PET) scaffolds mimicking in vivo microenvironment was developed. Using a modified microbioreactor platform designed specifically for 3-D cell cultures, three commonly used drugs, 5-fluorouracil (5-FU), gemcitabine, and sodium butyrate, were tested for their cytotoxicity on 3-D mouse embryonic stem (mES) cells and human colon cancer HT-29 cells, respectively. In general, 3-D cultures with multicellular structures exhibited similar expression in Ki-67 (a proliferation marker) and p27 $7^{\mathrm{kip} 1}$ (a quiescence marker) as compared to fresh tissues, and gave better predictive values of effective drug dosage in vivo. The 3-D multicellular mES and HT-29 cultures could be used as more reliable models for assessing dose-dependent drug responses, potentially reducing or partially replacing animal experiments, and thus should have applications in the early-stage drug discovery as well as toxicological evaluation of chemical compounds.
\end{abstract}

Keywords: Colon cancer cell; Cytotoxicity; Drug screening; Embryonic stem cell; High throughput; Microbioreactor 


\section{Introduction}

Drug discovery and development is a complex, lengthy and expensive process, in which the actual costs of discovering and developing a drug lie between $\$ 800$ million and $\$ 1.2$ billion with development timelines spanning 8-12 years [1]. Considerable costs are incurred later in the pipeline due to high failure rates in full clinical development (Phases IIb and III). The major cause of attrition in the clinical trials is lack of efficacy, which often cannot be reliably predicted because available screening models are notoriously lacking of predictive power [2, 3]. Historically, drug screening extensively relies on animal models as proxies for human beings in drug target validation and ADMET (absorption, distribution, metabolism, elimination and toxicity). Although these animal models have the capacity to provide a wealth of useful information for drug screening, they are relatively expensive, low throughput and ethically challenging. Therefore, there is growing demand for in vitro assays for drug screening and improvements in these assays that could significantly reduce the cost and time-to-market of new therapies $[2,4-6]$.

Currently, cell-based in vitro assays grow cells in two-dimensional (2-D) monolayer cultures as the predominant alternatives to animal models in primary drug screening [7]. However, 2-D cell-based assays cannot replicate in vivo counterparts and often cause discrepancy in predicting tissue specific responses [8-12]. Therefore, the current trend is to develop three-dimensional (3-D) cellular models that can better mimick in vivo tissues and give more reliable predictions on drug responses [13-17]. Various 3-D models for, such as, liver and skin have been developed [18-20]. However, it is challenging to monitor cell responses to drugs in 3-D cultures, which to date have been limited to end-point assay not suitable to high- 
throughput real-time dynamic drug pharmacokinetics studies [6]. Recently, we developed a label-free high-throughput fluorescence assay with cells expressing an enhanced green fluorescent protein (GFP) grown in a 3-D fibrous scaffold as an alternative in vitro method to study cytotoxicity and antineoplastic activity of drugs [12, 21]. This 3-D fluorescent cell-based assay could be used to screen potential drugs, such as Chinese herbal medicines, for their cytotoxicity and anti-cancer activity [22, 23].

In drug development, both efficacy and safety (cytotoxicity) of the drug must be screened. The goal of this study was to develop in-vitro tissue models for cytotoxicity assays with good predictive values on drug efficacy for screening cancer drugs. Mouse embryonic stem (mES) cells and colon cancer HT-29 cells were used to develop and demonstrate a working model for screening chemicals that are safe (with low or no cytotoxicity) and effective in treating colon cancer, the third most common cancer and the second leading cancer causing death, only next to lung cancer, in Western countries. Both the development of fetus and the existence of solid tumor are organized within a 3-D environment [24]. It is thus desirable to culture ES and colon cancer cells in 3-D scaffolds in developing a representative in-vitro tissue model for drug screening $[25,26]$. Three commonly used cancer drugs, 5-fluorouracil (5-FU), gemcitabine and sodium butyrate, were tested for their cytotoxicity effects on $\mathrm{mES}$ and human colon cancer cells. These chemicals were tested in 3 different models: 2-D monolayer model, 3-D low-density model, and 3-D high-density model with multicellular structures (tissue-like morphology). The results indicated that the 3-D multicellular model was more reliable in predicting tissue specific responses. This study demonstrated the use of an in vitro fluorescent HT-29 cell model to predict the efficacy and dosage of 5-FU for treating colon cancer.

\section{Materials and Methods}




\subsection{Chemicals}

Both 5-fluorouracil (5-FU) and sodium butyrate were purchased from Sigma Chemical Company (St. Louis, MO), while gemcitabine was obtained from Eli Lilly Co. (Indianapolis, IN).

\subsection{EGFP Cell Lines and Media}

Murine embryonic stem (mES) D3 cells (ATCC CRL-1934) and human colon cancer HT-29 cells (ATCC HTB-38) stably expressing enhanced green fluorescence protein (EGFP) were obtained by transfection with plasmid pEGFP-N3 (Clontech, Mountain View, CA) using Lipofectamine 2000 (Invitrogen, Grand Island, NY), following the method previously described $[12,23]$. After an initial 10-day screening using geneticin (G418, Gibco, Grand Island, NY), EGFP expressing colonies were isolated under a fluorescent microscope and then cells were subcultured in the absence of the selective pressure every 5 days for 20 passages. The stability of the transfected cell lines were verified (>97\%) using FACS Calibur (BD Biosciences, San Jose, CA). EGFP expression was controlled with the human cytomegalovirus (CMV) promoter [27], a constitutive promoter giving stable and high expression of the fluorescence protein in proliferating mES and HT-29 cells $[12,21]$.

Unless otherwise noted, ES D3 and ES-GFP cells were maintained on gelatin pre-coated T-flasks containing Dulbecco's modified Eagle's medium (DMEM, high glucose 4.5 g/L, Invitrogen, Grand Island, NY) supplemented with $10 \%$ fetal bovine serum (FBS, Invitrogen), $100 \mathrm{U} / \mathrm{mL}$ penicillin (Invitrogen), $100 \mu \mathrm{g} / \mathrm{mL}$ streptomycin (Invitrogen), $2 \mathrm{mM}$ L-glutamine (Invitrogen), $100 \mu \mathrm{M}$ monothioglycerol (Sigma-Aldrich) and $1000 \mathrm{U} / \mathrm{mL}$ leukemia inhibitory factor (LIF, Millipore, Billerica, MA). Both ES-GFP and its parental strain maintained a high stage-specific embryonic antigen (SSEA-1) expression level $(>95 \%)$ in the aforementioned medium. HT-29 and HT-29-GFP were cultured in DMEM (high glucose $4.5 \mathrm{~g} / \mathrm{L}$, Gibco) 
supplemented with $10 \%$ FBS. These cultures were incubated in a humidified cell culture incubator at $37^{\circ} \mathrm{C}$ with $5 \% \mathrm{CO}_{2}$.

\subsection{Cytotoxicity Assays in 2-D Cultures}

Each well on the 12-well plates was seeded with 50,000 cells, which were then exposed to chemicals one day after inoculation. Cell number in each well was quantified daily with Trypan Blue (Sigma-Aldrich) staining using a hemocytometer. $\mathrm{IC}_{50}$ was calculated as the concentration of chemicals resulting in $50 \%$ reduction in the viable cell density as compared to the control on day 3 for ES cells and day 6 for HT-29 cells.

\subsection{Cytotoxicity Assays in 3-D Cell Cultures}

Unless otherwise noted, cells were cultured in nonwoven polyethylene terephthalate (PET) fibrous disks (fiber diameter: $\sim 20 \mu \mathrm{m}$; disk diameter: $6.2 \mathrm{~mm}$; disk thickness: $1 \mathrm{~mm}$ ) in modified 96-well plates, each with 6 microbioreactors (working volume: $3 \mathrm{~mL}$ ), each of which consisted of 9 original wells with their walls removed, as described in our previous studies [12, 22]. Briefly, PET matrices were soaked in $10 \mu \mathrm{g} / \mathrm{mL}$ fibronectin for $12 \mathrm{~h}$ and then in the growth medium for another $12 \mathrm{~h}$. After removing medium, each PET scaffold in a 96-well plate was seeded with 25,000 ES-GFP cells or 40,000 HT-29-GFP cells in $25 \mu 1$ medium. The 96-well plate was put into a $\mathrm{CO}_{2}$-incubator for $6 \mathrm{~h}$, allowing cells to attach to the fibrous matrix, and 180 $\mu \mathrm{L}$ of growth medium were then added to each well and incubated for another $24 \mathrm{~h}$. Then, each cell-containing scaffold was transferred gently with a sterile tweezer to the modified 96-well plate for 3-D cytotoxicity studies. The cytotoxicity assay was also performed in a modified 384well plate with 40 microbioreactors (Fig. 1), each with a 1-mL working volume [21]. Unless otherwise noted, 10,000 (ES-GFP) or 16,000 (HT-29-GFP) cells in $11 \mu 1$ medium were seeded into each pretreated scaffold, which was then placed in the microbioreactor containing $1 \mathrm{~mL}$ 
medium. The multi-well plates were stacked onto an orbital shaker (Belly Button Shaker, Stovall, Greensboro, $\mathrm{NC}$ ) at a rotational speed of $60 \mathrm{rpm}$ in a $\mathrm{CO}_{2}$-incubator. Chemicals including 5-FU, sodium butyrate and gemcitabine at different concentrations were added to the cultures 1 day (3D low density models), 4 days (3-D high density ES-GFP model), or 8-10 days (3-D high density HT-29-GFP model) after inoculation. To measure the culture fluorescence, the plate was transferred to a fluorescent plate reader (GENios Pro, Tecan, Durham, NC) at $37^{\circ} \mathrm{C}$, with bottom reading as the measurement mode. All assays were conducted in triplicate or duplicate. Cell proliferation was estimated from fluorescence signal, which was proportional to the number of cells present in the 3-D PET matrix $[12,21]$. IC $_{50}$ was calculated as the concentration of chemicals resulting in 50\% reduction in the peak fluorescence signal as compared to the control.

\subsection{Immunohistochemistry of Cell Cycle Biomarkers}

To evaluate the cell cycle progression of HT-29 cells in 3-D scaffolds and 2-D monolayer cultures, a cell proliferation marker Ki-67 and a quiescence marker $\mathrm{p} 27^{\mathrm{kip} 1}$ were studied using immunohistochemistry. Fresh human tonsil cells were used as control. Agarose-embedded cell pellets were prepared for histological processing of cells from 2-D monolayer cultures (1 week) as follows: 1) freshly trypsinized $10^{7}$ cells were spun down into a pellet followed by supernatant removal; 2) cells were then re-suspended by vortexing at a maximum speed; 3) at the same time, 3\% agarose solution in PBS was prepared in a microwave; 4) after the agarose solution was cooled down to about $50{ }^{\circ} \mathrm{C}$, cells were added at an equivalent volume and mixed immediately; 5) the agarose-embedded cell pellet was cooled down at $-20^{\circ} \mathrm{C}$ for $10 \mathrm{~min}$ before fixing.

Fresh human tonsil, 3-D tissue cultures (2 weeks) and agarose-embedded cell pellets were fixed in $10 \%$ formalin, embedded in paraffin, and then horizontally cut into $4 \mu \mathrm{m}$ thick sections. For immunohistochemical staining, the sections were placed on positively charged 
slides, and then dewaxed and rehydrated with xylenes and graded alcohols. Slides were peroxidized with $3 \% \mathrm{H}_{2} \mathrm{O}_{2}$ for 5 min to inhibit endogenous peroxidase before antigen retrieval. Slides were then placed on an immunostaining system (Dako Autostainer). After washing with PBS, primary antibody against Ki-67 (1:150 in PBS; Dako, M7203) or p27 ${ }^{\text {kip1 }}$ (1:50 in PBS; Dako M7240) was added at room temperature, followed with consecutive additions of biotinylated antibody, enzyme conjugated streptavidin, and substrate chromogen DAB. Slides were then counterstained with hematoxylin, dehydrated with graded alcohols, and cover slipped.

\subsection{Statistical Analysis}

Unless otherwise noted, all assays were done with triplicate samples. A one-way analysis of variance (ANOVA) was performed using JMP version 7.0, with $p<0.05$ as the threshold for significant difference.

\section{Results}

\subsection{2-D Cultures}

The cytotoxicity of 5-FU, gemcitabine and sodium butyrate were apparent in 2-D cultures of ES and HT-29 cells as the final viable cell density decreased with increasing the chemical concentration (Table 1). The $\mathrm{IC}_{50}$ values or the concentrations of the chemicals resulting in $50 \%$ reduction in the viable cell density as compared to the control for ES and HT-29 cells were estimated from the data and are listed in Table 2. In general, the 2-D cultures showed that ES cells were more sensitive to these drugs as indicated by its lower IC $_{50}$ values compared to HT-29 cells.

\subsection{3-D Low-density Cultures}


It is difficult to directly count cell number in 3-D cultures. However, cells emitting the green fluorescent light can be quantified by monitoring the fluorescent signals (see Fig. 2A, B), which can be used to evaluate drug effects on ES and HT-29 cells. Fig. 3 shows the time course profiles of culture fluorescence of ES and HT-29 cells in 3-D cultures as affected by 5-FU, gemcitabine and sodium butyrate at various concentrations. ES cell growth decreased with increasing the 5-FU concentration and was completely inhibited at $\sim 0.62 \mu \mathrm{M}$ (Fig. 3A). However, 5-FU did not show obvious cytotoxicity on HT-29 cells even at $2 \mu \mathrm{M}$ (Fig. 3D). Gemcitabine completely inhibited ES cell growth at $0.025 \mu \mathrm{M}$, but seemingly stimulated ES cell growth (based on the higher culture fluorescence intensities) at lower concentrations (Fig. 3B). Compared to the control, increasing gemcitabine from 0.00004 to $0.001 \mu \mathrm{M}$ also increased cell growth rate, although cell growth stopped earlier at a lower maximum density. For HT-29 cells, gemcitabine at $0.005 \mu \mathrm{M}$ showed strong growth inhibition effect that increased with increasing the dosage, although gemcitabine initially increased culture fluorescence compared to the control (Fig. 3E). The increased culture fluorescence by gemcitabine, mainly at the initial exponential phase of the culture, suggested that gemcitabine at low concentrations increased GFP expression, probably through its effect on the CMV promoter, whose activity could be affected by external stimuli and enhancers such as sodium butyrate [28, 29]. Sodium butyrate, a widely used enhancer for protein expression [30], at low concentrations (0.04-0.2 mM for ES cells and 0.5-2 mM for HT-29 cells) significantly increased culture fluorescence (Fig. 3C, 3F), because of increased expression of green fluorescence protein. Sodium butyrate inhibited ES cell growth at $1 \mathrm{mM}$ and HT-29 cell growth at $8 \mathrm{mM}$. These results demonstrated dose-sensitive responses of GFP expression (and cell growth) to these chemicals. 
Based on these 3-D culture data on the peak fluorescence signal reached in each condition, the $\mathrm{IC}_{50}$ values were estimated and are compared to the $\mathrm{IC}_{50}$ values from the 2-D cultures (Table 2). Except for 5-FU on HT-29 cell, comparable IC $_{50}$ values (on the same order of magnitude) were obtained for both 2-D and 3-D low-density cultures, although 3-D cultures generally showed higher $\mathrm{IC}_{50}$ values, suggesting cells in 3-D cultures were less sensitive to drugs as compared to 2-D cultures. Interestingly, the $\mathrm{IC}_{50}$ values of 5-FU for HT-29 cell in the 3-D culture was two orders of magnitude higher than that in 2-D culture, suggesting that colon cancer cells would be more drug-resistant in the 3-D environment. Similar finding has also been reported for breast cancer MCF-7 cells, which showed 10-fold higher $\mathrm{IC}_{50}$ when grown in 3-D scaffolds than on 2-D surface [31].

It should be noted that cells in 2-D monolayer cultures usually would have a shorter doubling time due to increased cell contact and stretching on a plenary surface, which can enhance proliferation [32]. However, the apparent doubling time for HT-29-GFP cells was longer in 2-D cultures $(\sim 24 \mathrm{~h})$ than in 3-D cultures $(\sim 17 \mathrm{~h})$, probably because cell growth in the 2-D cultures was limited by confluence on the available surface.

\subsection{3-D High-density Cultures with Tissue-like Morphology}

Cytotoxicity assays in 3-D high-density cultures were performed with cells precultured to a high density forming large cell aggregates (see Fig. 2C, D) before exposure to chemicals. Fig. 4 shows the responses of ES and HT-29 cells to 5-FU, gemcitabine and sodium butyrate in 3-D high-density cultures. ES cells present in the high-density cultures were not sensitive to or inhibited by 5-FU up to $77 \mu \mathrm{M}$ (Fig. 4A) and gemcitabine up to $10 \mu \mathrm{M}$ (Fig. 4B). Even at the highest concentrations (770 $\mu \mathrm{M}$ 5-FU and $100 \mu \mathrm{M}$ gemcitabine) tested, only minimal cytotoxicity effect could be detected. On the other hand, sodium butyrate showed strong 
inhibition on ES cells at $1 \mathrm{mM}$ and completely inhibited growth and caused cell death at $10 \mathrm{mM}$ (Fig. 4C), which was consistent with a previous report that sodium butyrate at $3 \mathrm{mM}$ or higher would cause apoptosis and significantly decrease the specific growth rate of ES cells [21].

For HT-29 cells in the high-density culture, 5-FU exhibited a dose-dependent inhibition effect and caused early cell death at $770 \mu \mathrm{M}$ (Fig. 4D), whereas gemcitabine exhibited limited cytotoxicity at concentrations lower than $1000 \mu \mathrm{M}$ (Fig. 4E). Similar to the low-density cultures, sodium butyrate at low concentrations $(0.1-10 \mathrm{mM})$ appeared to increase GFP expression in HT29 cells in the high-density culture (Fig. 4F). However, at the higher concentrations of $100 \mathrm{mM}$ and $500 \mathrm{mM}$, sodium butyrate showed acute cytotoxicity on HT-29 cells and caused immediate cell death upon drug addition.

Based on the drug response data, the $\mathrm{IC}_{50}$ values of the high-density cultures were estimated and are also listed in Table 2 for comparison. Both mESC and HT-29 cells were highly sensitive to gemcitabine in 2-D monolayer and 3-D low-density cultures, as indicated by their relatively low $\mathrm{IC}_{50}$ values. However, they showed much higher drug resistance when present with multicellular structures in 3-D high-density cultures, with $\mathrm{IC}_{50}$ values increased by several orders of magnitude $\left(10^{2}\right.$ to $\left.10^{5}\right)$ (Table 2). The effect of multicellular structures on increased drug resistance can be reflected by the resistance factor ( $\mathrm{Rf}$ ) or the ratio of $\mathrm{IC}_{50}$ for the 3-D high-density culture to the $\mathrm{IC}_{50}$ for the 2-D or 3-D low-density cultures. Surprisingly, the presence of multicellular structures in the high-density cultures did not show any effect on the cytotoxicity of sodium butyrate for ES-GFP cells $(\mathrm{Rf}=1)$, although the Rf value for HT-29 cells was higher at $\sim 30$.

In 3-D high-density cultures, ES-GFP cells showed an extremely high resistance to 5-FU $\left(\mathrm{IC}_{50}>770 \mu \mathrm{M}\right)$, while 5-FU at $770 \mu \mathrm{M}$ was effective in killing colon cancer (HT-29) cells. 
However, 5-FU exhibited no cytotoxic effect on 3-D high-density ES cell model. This selective cytotoxicity toward colon cancer cells is consistent with the fact that 5-FU has the greatest impact on colon cancer and remains the most popular therapeutic agent for about half a century. However, this selective cytotoxicity effect could not be predicted in 2-D monolayer cultures, which showed that ES-GFP cells were more sensitive to 5-FU with an $\mathrm{IC}_{50}$ lower than that for HT-29-GFP. The dramatic different drug responses of cells in 2-D monolayer and 3-D highdensity cultures could be attributed to the difference in cell physiology or cell cycle stage, as shown in the immunohistochemistry study discussed below.

\subsection{Immunohistochemistry Characterization of Cell Cycle Stage}

As shown in Fig. 5, the fraction of Ki-67 positive cells was low (10\%-20\%) in both fresh human tonsil tissue and cell aggregates from 3-D high-density cultures, while a much higher proportion (30\%-40\%) of cells from 2-D monolayer cultures was Ki-67 positive. In contrast, a significantly higher proportion of $\mathrm{p} 27^{\mathrm{kip} 1}$ positive cells $(30 \%-40 \%)$ was observed in fresh human tonsil tissue and 3-D cultured tissues as compared to a very low percentage $(<5 \%)$ of quiescent cells in the monolayer cultures. Ki-67 antigen is present in all proliferating cells, which are at the active phases of cell cycle (G1, S, G2, and M phases), but is absent in resting cells (G0). Therefore, it is an excellent biomarker for proliferating cells [33] and a good indicator of cell responses to chemotherapy in aggressive tumors $[34,35]$. In contrast, $\mathrm{p} 27^{\mathrm{Kip} 1}$ can inhibit cyclindependent kinases (CDKs) by binding to cyclin/CDK complexes and modulate the transition from G1 to S. Furthermore, p2 $7^{\text {Kip1 }}$ can induce growth arrest, differentiation and apoptosis [36]. Cell proliferation and quiescence indicated by immunohistochemical staining of Ki-67 and p2 $7^{\text {kip1 }}$ suggested that 3-D multicellular model had similar cell cycle progression as human solid tissues and would be a more accurate model of the complex in vivo microenvironments as 
compared to conventional 2-D cultures.

\section{Discussion}

Conventional drug discovery and pharmaceutical research usually involve cell-based assays and animal experiments. However, cell-based assays with conventional 2-D monolayer cultures often failed to predict drug responses in vivo, while animal experiments present moral issues as well as high costs. Therefore, the development of alternative in vitro cell-based assays is in urgent need [2]. High-throughput multi-cellular assays could serve as a bridge between monolayer cultures and animals to remove poor candidates and increase pipeline efficiency in drug discovery and at the same time discover the mechanism of drug actions in pharmacological studies [6].

In the present study, we showed that the drug effect might be dramatically different and the selectivity of drug to different cell types could be totally opposite in different in vitro models with 2-D monolayers and 3-D multicellular structures. In general, cells, both normal and tumor (as abnormal), are highly proliferating when grown in 2-D monolayer cultures. However, except for developing organs, most normal somatic cells in vivo stop proliferating and stay in a quiescent state, which could cause a high-level resistance to antineoplastic drugs [37, 38]. The percentage of rapidly dividing cells in tumors is also low, although it could be higher than that of normal cells. In addition to drug absorption, penetration and metabolism, the high percentage of quiescent cells could result in resistance of chemotherapeutic agents, which could not be revealed by highly proliferating 2-D monolayer cultures [4]. Consequently, 2-D cultures would not be a good in vitro model for screening cancer drugs, although they may be suitable, and have been widely used, for screening anti-metabolic agents, which are actively acting against proliferating cells but not quiescent cells. 
Compared to normal cells (e.g., ES cells), solid tumor cells are usually more resistant to toxic chemical agents, as demonstrated with sodium butyrate and gemcitabine in both 2-D monolayer and 3-D cultures in this study. However, some cancer drugs, such as 5-FU, have good selectivity for inhibiting/killing tumor cells over normal tissues, which might not be revealed in 2-D monolayer or even 3-D low-density cultures. Tumor cells come from mutations, which could activate oncogenes or repress tumor suppressor. These mutations could prevent cells from apoptosis even when cellular DNA is damaged severely. Therefore, tumor cells could be more resistant to genotoxic substances as compared to normal cells, especially when cultured in monolayers [39]. On the other hand, although chemotherapy has been demonstrated with cytotoxic effects on healthy cells in vivo, most normal cells except for those with a higher replacement or proliferation rate are more resistant to chemotherapy than tumor cells.

In the present study, we developed high-density multicellular tissue-like models that can more accurately predict the required dosage $(77-770 \mu \mathrm{M})$ of 5-FU for treating colon cancer (HT29 cell model) yet not harmful to normal tissue (mES cell model). 5-FU has been the most commonly used chemotherapeutic agent against colon cancer during the past few decades and new treatments combining 5-FU with other drugs are continually emerging [40]. Both the reported peak plasma concentrations of 5-FU $(385 \mu \mathrm{M})$ [41] and the achieved serum concentration after in vivo administration $(77 \mu \mathrm{M})$ [42] were much higher than $\mathrm{IC}_{50}$ from 2-D HT-29 cultures but comparable to that from 3-D high-density HT-29 cultures. Clearly, the 3-D high-density culture models developed in this study could replicate in vivo human biology more accurately and provide a better predictive value, whereas assays based on 2-D cultures were erroneous in determining effective drug dosages. It should be noted that the 3-D multicellular model also correctly predicted that 5-FU had a higher efficacy than gemcitabine in killing colon 
cancer cells, which agreed with clinical results showing the low performance of gemcitabine and the greatest effectiveness of 5-FU in colon cancer treatment. In contrast, the 2-D HT-29 model incorrectly suggested that gemcitabine would be the more effective drug than 5-FU in treating colon cancer. It has also been shown that some drugs such as PX-866 suppressed cancer cells in 3-D spheroids but not cells grown on 2-D plastic surface [43]. Clearly, 2-D monolayer cultures are not good models for predicting drug efficacy.

There were significant discrepancies between the experimental results from conventional 2-D cell-based assays and those from clinical trials while using sodium butyrate and gemcitabine in treating colorectal cancers $[44,45]$. These discrepancies may be because conventional 2-D cultures cannot mimic the complex cellular heterogeneity and interactions and in vivo tumor microenvironments $[6,17]$. On the other hand, 3-D high-density culture models with tissue-like structures accurately predicted that 5-FU was more effective in inhibiting colon cancer than gemcitabine. Sodium butyrate showed good efficacy in treating HT-29 colon cancer in both 3-D low-density and 3-D high-density (tissue-like) models. However, it failed in clinical trials because of organoleptic problem and rapid blood elimination in vivo, which could not be represented in the present model. The development of organs-on-chips and human-on-a-chip would be necessary for systematic drug assessments [46, 47].

The fluorescent cells used in the 3-D multicellular model can provide a highly automated, real-time monitoring of cell responses to drugs in a high-throughput dynamic manner. However, the culture fluorescent signals can be affected by both the total cell number and the expression level of GFP in each cell, which could also be affected by the drug in a dosage-dependent manner. As discussed before, 5-FU, sodium butyrate, and gemcitabine at low concentrations significantly increased culture fluorescence, mainly because of an increase in the cell specific 
fluorescence, instead of the total cell number, as a result of increased cell size and the specific expression of GFP caused by the drug. In fact, we found a strong correlation between the cell specific fluorescence and cell size (data not shown). Due to the S-phase specificity of the CMV promoter used in driving GFP expression, chemicals inducing S-phase arrest, such as 5-FU and gemcitabine, could increase cell size and cell specific fluorescence. It has been shown that the percentage of colon cancer cells in S-phase increased from $57 \%$ to $87 \%$ after treating with 38.5 $\mu \mathrm{M}$ 5-FU for $24 \mathrm{~h}$ [48]. In addition, some chemicals, such as butyrate, could cause G0/G1 arrest and increase cell specific productivity of heterologous protein. The increased cell specific fluorescence with drug addition confounded the culture fluorescence based estimation of cell number, which could be an impediment in fluorescence toxicity tests, especially in 2-D and 3-D low-density cultures. On the other hand, the increased fluorescence intensity reflected well the cell viability and its ability to produce cell constituents (protein) and to grow in size, which cannot be detected when only cell number was counted with the conventional method. In this regard, the fluorescent signal might actually be a better measurement of cell viability than cell number. Furthermore, variation in the cell specific fluorescence should not be a concern in 3-D high-density cultures as the effective drug dosage would be much higher, and therefore, the dynamic responses in culture fluorescence to drugs in high-density 3-D cultures could be reliably used to predict effective or lethal dosages required to treat cancer as demonstrated in this study. Nevertheless, other strong constitutive promoters, such as EF1A and CAGG, with consistent expression levels in a wide range of mammalian cell lines should also be explored in future studies $[27,49]$.

\section{Conclusions}


Conventional 2-D proliferation assays cannot correctly predict the efficacy of drugs, because 2-D cell cultures in multi-well plates cannot represent in vivo tissues that are present in 3-D environments. Therefore, cell-based assays with multicellular structures are desired in drug screening and discovery to give a better predictive value than monolayer cultures. In this study, ES and HT-29 cell aggregates with in vivo likeness cultured in 3-D fibrous scaffolds in microbioreactor array were used for drug screening. The 3-D multicellular models can better resemble in vivo tissue status and provide temporal responses in a dynamic high-throughput manner. Compared to 2-D monolayer cultures, the 3-D tissue-like model can give a much better predictive value for hits to enter in vivo tests, thus reducing or partially replacing expensive and time-consuming animal experiments in the drug discovery process. The 3-D cell-based platform thus should have important application for anticancer drug screening.

\section{Acknowledgements}

This work was supported in part by the National Science Foundation Nanoscale Science and Engineering Center (EEC-0425626).

\section{References}

[1] D.W. Grainger, Cell-based drug screening; this world is not flat, Adv. Drug Del. Rev. 6970(2014) vii-xi.

[2] A. Astashkina, D.W. Grainger, Critical analysis of 3-D organoid in vitro cell culture models for high-throughput drug candidate toxicity assessments, Adv. Drug Del. Rev. 69-70(2014) $1-18$.

[3] I. Kola, J. Landis, Can the pharmaceutical industry reduce attrition rates? Nature Rev. 3(2004) 711-715. 
[4] J.L. Horning, S.K. Sahoo, S. Vijayaraghavalu, S. Dimitrijevic, J.K. Vasir, T.K. Jain, A.K. Panda, V. Labhasetwar, 3-D tumor model for in vitro evaluation of anticancer drugs, Mol. Pharm. 5(2008) 849-862.

[5] C.R. Thoma, M. Zimmermann, I. Agarkova, J.M. Kelm, W. Krek, 3D cell culture systems modeling tumor growth determinants in cancer target discovery, Adv. Drug Del. Rev. 6970(2014) 29-41.

[6] R. Zang, D. Li, I.C. Tang, J. Wang, S.T. Yang, Cell-based assays in high-throughput screening for drug discovery. Int. J. Biotechnol. Wellness Ind. 1(2012) 31-51.

[7] S. Zhang, Beyond the Petri dish, Nat. Biotechnol. 22(2004) 151-152.

[8] K. Bhadriraju, C.S. Chen, Engineering cellular microenvironments to improve cell-based drug testing, Drug Discov. Today 7(2002) 612-620.

[9] R. Derda, A. Laromaine, A. Mammoto, S. Tang, T. Mammoto, D.E. Ingber, G.M. Whitesides, Paper-supported 3D cell culture for tissue-based bioassays, Proc. Natl. Acad. Sci. USA 106 (2009) 18457-18462.

[10] J. Friedrich, R. Ebner, L.A. Kunz-Schughart, Experimental anti-tumor therapy in 3-D: spheroids--old hat or new challenge? Int. J. Radiat. Biol. 83(2007) 849-871.

[11] K.R. Laderoute, B.J. Murphy, S.M. Short, T.D. Grant, A.M. Knapp, R.M. Sutherland, Enhancement of transforming growth factor-alpha synthesis in multicellular tumour spheroids of A431 squamous carcinoma cells, Br. J. Cancer 65(1992) 157-162.

[12] X. Zhang, S.T. Yang, High-throughput 3-D cell-based proliferation and cytotoxicity assays for drug screening and bioprocess development, J. Biotechnol. 151(2011) 186-193.

[13] N.T. Elliott, F. Yuan, A review of three-dimensional in vitro tissue models for drug discovery and transport studies, J. Pharma. Sci. 100(2011) 59-74.

[14] L.G. Griffith, M.A. Swartz, Capturing complex 3D tissue physiology in vitro, Nat. Rev. Mol. Cell Biol. 7(2006) 211-224.

[15] J.A. Hickman, R. Graeser, R. de Hoogt, S. Vidic, C. Brito, M. Gutekunst, H. van der Kuip, Three-dimensional models of cancer for pharmacology and cancer cell biology: Capturing tumor complexity in vitro/ex vivo, Biotechnol J. 9(2014) 1115-1128.

[16] D.M. Timm, J. Chen, D. Sing, J.A. Gage, W.L. Haisler, S.K. Neeley, R.M. Raphael, M. Dehghani, K.P. Rosenblatt, T.C. Killian, H. Tseng, G.R. Souza, A high-throughput three- 
dimensional cell migration assay for toxicity screening with mobile device-based macroscopic image analysis, Sci. Reports 3(2013) 3000.

[17] S.T. Yang, X. Zhang, Y. Wen, Microbioreactors for high-throughput cytotoxicity assays, Curr. Opin. Drug Discov. Devel. 11(2008) 111-127.

[18] M. Bokhari, R.J. Carnachan, N.R. Cameron, S.A. Przyborski, Culture of HepG2 liver cells on three dimensional polystyrene scaffolds enhances cell structure and function during toxicological challenge, J. Anat. 211(2007) 567-576.

[19] I. Canton, D.M. Cole, E.H. Kemp, P.F. Watson, J. Chunthapong, A.J. Ryan, S. MacNeil, J.W. Haycock, Development of a 3D human in vitro skin co-culture model for detecting irritants in real-time, Biotechnol. Bioeng. 106(2010) 794-803.

[20] I. Wagner, E.M. Materne, S. Brincker, U. Sussbier, C. Fradrich, M. Busek, F. Sonntag, D.A. Sakharov, E.V. Trushkin, A.G. Tonevitsky, R. Lauster, U. Marx, A dynamic multiorganchip for long-term cultivation and substance testing proven by 3D human liver and skin tissue co-culture, Lab Chip 13(2013) 3538-3547.

[21]R. Zang, X. Zhang, M. Li, S.T. Yang, Microwell bioreactor system for cell-based high throughput proliferation and cytotoxicity assays, Process Biochem. 48(2013) 78-88.

[22] D. Li, R. Zang, S.T. Yang, J. Wang, X. Wang, Cell-based high-throughput proliferation and cytotoxicity assays for screening traditional Chinese herbal medicines, Process Biochem. 48(2013) 517-524.

[23] D. Li, S. Isherwood, A. Motz, R. Zang, S.T. Yang, J. Wang, X. Wang, Cell-based screening of traditional Chinese medicines for proliferation enhancers of mouse embryonic stem cells, Biotechnol. Prog. 29(2013) 738-744.

[24] C. Godugu, A.R. Patel, U. Desai, T. Andey, A. Sams, M. Singh, AlgiMatrix ${ }^{\text {TM }}$ based 3D cell culture system as an in-vitro tumor model for anticancer studies, PLoS One 8(2013) e53708.

[25] E. Cukierman, R. Pankov, D.R. Stevens, K.M. Yamada, Taking cell-matrix adhesions to the third dimension, Science 294(2001) 1708-1712.

[26] F. Pampaloni, E.G. Reynaud, E.H.K. Stelzer, The third dimension bridges the gap between cell culture and live tissue, Nat. Rev. Mol. Cell Biol. 8(2007) 839-845. 
[27] J.Y. Qin, L. Zhang, K.L. Clift, I. Hulur, A.P. Xiang, B.Z. Ren, B.T. Lahn, Systematic comparison of constitutive promoters and the doxycycline-inducible promoter, PLoS One 5(2010) e10611. doi:10.1371/journal.pone.0010611

[28] L. Hunt, M. Jordan, M. De Jesus, F.M. Wurm, GFP-expressing mammalian cells for fast, sensitive, noninvasive cell growth assessment in a kinetic mode, Biotechnol. Bioeng. 65(1999) 201-205.

[29]X. Zhang, S.T. Yang, An online, non-invasive fluorescence probe for immobilized cell culture process development, Process Biochem. 46(2011) 2030-2035.

[30] K.H. Chang, K.S. Kim, N-acetylcysteine increases the biosynthesis of recombinant EPO in apoptotic Chinese Hamster Ovary cells. Free Radic. Res. 30(1999) 85-91.

[31] H.K. Dhiman, A.R. Ray, A.K. Panda, Three-dimensional chitosan scaffold-based MCF-7 cell culture for the determination of the cytotoxicity of tamoxifen, Biomaterials 26(2005) 979-986.

[32] C.M. Nelson, C.S. Chen, Cell-cell signaling by direct contact increases cell proliferation via a PI3K-dependent signal, FEBS Lett. 514(2002) 238-242.

[33] J. Gerdes, Ki-67 and other proliferation markers useful for immunohistological diagnostic and prognostic evaluations in human malignancies, Semin. Cancer Biol. 1(1990) 199-206.

[34] S. Kamoi, Y. Ohaki, S. Okada, N. Matsushita, T. Kawamura, T. Araki, Mitotic index and ki67 nuclear antigen labeling index as predictors of chemotherapy response in uterine cervical carcinoma, Gynecol. Oncol. 83(2001) 555-559.

[35] T. Petit, M. Wilt, M. Velten, R. Millon, J.F. Rodier, C. Borel, R. Mors, P. Haegele, M. Eber, J.P. Ghnassia, Comparative value of tumour grade, hormonal receptors, Ki-67, HER-2 and topoisomerase II alpha status as predictive markers in breast cancer patients treated with neoadjuvant anthracycline-based chemotherapy, Eur. J. Cancer 40(2004) 205-211.

[36] A. Sgambato, A. Cittadini, B. Faraglia, I.B. Weinstein, Multiple functions of p27(Kip1) and its alterations in tumor cells: a review, J. Cell Physiol. 183(2000) 18-27.

[37] R.C. Jackson, The problem of the quiescent cancer cell, Adv. Enzyme Regul. 29(1989) 2746. 
[38] H.R. Mellor, D.J.P. Ferguson, R. Callaghan, A model of quiescent tumour microregions for evaluating multicellular resistance to chemotherapeutic drugs, Br. J. Cancer 93(2005) 302309.

[39]E. Behravesh, K. Emami, H. Wu, S. Gonda, Comparison of genotoxic damage in monolayer cell cultures and three-dimensional tissue-like cell assemblies, Adv. Space Res. 35(2005) 260-267.

[40] M. Ducreux, A. Adenis, J.P. Pignon, E. Francois, B. Chauffert, J.L. Ichante, E. Boucher, T. Conroy, Efficacy and safety of bevacizumab-based combination regimes in patients with previously untreated metastatic colorectal cancer, Eur. J. Cancer 49(2013) 1236-1245.

[41] D.S. Alberts, H.S. Chen, Tabular summary of pharmacokinetic parameters relevant to in vitro drug assay, Prog. Clin. Biol. Res. 48(1980) 351-359.

[42] R. Grantab, S. Sivananthan, Ian F. Tannock, The penetration of anticancer drugs through tumor tissue as a function of cellular adhesion and packing density of tumor cells, Cancer Res. 66(2006) 1033-1039.

[43] A.L. Howes, G.G. Chiang, E.S. Lang, C.B. Ho, The phosphatidylinositol 3-kinase inhibitor, PX-866, is a potent inhibitor of cancer cell motility and growth in three-dimensional cultures, Mol. Cancer Ther. 6(2007) 2505-2514.

[44] P.R. Pouillart, Role of butyric acid and its derivatives in the treatment of colorectal cancer and hemoglobinopathies, Life Sci. 63(1998) 1739-1760.

[45]E. Smitskamp-Wilms, H.M. Pinedo, G. Veerman, V.W.T.R. Van Haperen, G.J. Peters, Postconfluent multilayered cell line cultures for selective screening of gemcitabine, Eur. J. Cancer 34(1998) 921-926.

[46] D. Huh, H.J. Kim, J.P. Fraser, D.E. Shea, M. Khan, A. Bahinski, G.A. Hamilton, D.E. Ingber, Microfabrication of human organs-on-chips, Nat. Protoc. 8(2013) 2135-2157.

[47] U. Marx, H. Walles, S. Hoffmann, G. Lindner, R. Horland, F. Sonntag, U. Klotzbach, D. Sakharov, A. Tonevitsky, R. Lauster, 'Human-on-a-chip' developments: a translational cutting-edge alternative to systemic safety assessment and efficiency evaluation of substances in laboratory animals and man? Altern. Lab. Anim. 40(2012) 235-257.

[48] R.A. Bras-Goncalves, M. Pocard, J.L. Formento, F. Poirson-Bichat, G. De Pinieux, I. Pandrea, F. Arvelo, G. Ronco, P. Villa, A. Coquelle, Synergistic efficacy of n-butyrate and 
5-fluorouracil in human colorectal cancer xenografts via modulation of DNA synthesis, Gastroenterology 120(2001) 874-888.

[49] C. Teschendorf, K.H. Warrington, Jr. D.W. Siemann, N. Muzyczka, Comparison of the EF1.a and the CMV promoter for engineering stable tumor cell lines using recombinant adenoassociated virus, Anticancer Research 22(2002) 3325-3330. 
Table 1. Effects of 5-FU, gemcitabine and sodium butyrate on ES-GFP and HT-29-GFP cell growth in 2-D cultures at 3 and 6 days, respectively.

\begin{tabular}{lcccc}
\hline & \multicolumn{2}{c}{ ES cells } & \multicolumn{2}{c}{ Colon cancer cells } \\
\cline { 2 - 5 } & Dose & $\begin{array}{c}\text { Viable cell density } \\
\left(10^{4} \text { cells } / \mathrm{mL}\right)\end{array}$ & Dose & $\begin{array}{c}\text { Viable cell density }^{\#} \\
\left(10^{4} \text { cells } / \mathrm{mL}\right)^{*}\end{array}$ \\
\hline \multirow{2}{*}{ 5-FU } & 0 & 133 & $0 \mu \mathrm{M}$ & 320 \\
& $0.096 \mu \mathrm{M}$ & 120 & $2 \mu \mathrm{M}$ & 145 \\
& $0.384 \mu \mathrm{M}$ & 30 & $10 \mu \mathrm{M}$ & 103 \\
\hline \multirow{2}{*}{ Gemcitabine } & $1.73 \mu \mathrm{M}$ & 16 & $50 \mu \mathrm{M}$ & 18 \\
& 0 & 133 & $0 \mu \mathrm{M}$ & 294 \\
& $0.001 \mu \mathrm{M}$ & 60 & $0.001 \mu \mathrm{M}$ & 254 \\
& $0.01 \mu \mathrm{M}$ & 20 & $0.005 \mu \mathrm{M}$ & 140 \\
\hline \multirow{2}{*}{ Sodium Butyrate } & $0.1 \mu \mathrm{M}$ & 2 & $0.025 \mu \mathrm{M}$ & 14 \\
& 0 & 133 & $0 \mathrm{mM}$ & 260 \\
& $2 \mathrm{mM}$ & 8 & $0.5 \mathrm{mM}$ & 259 \\
& $32 \mathrm{mM}$ & 0 & $2 \mathrm{mM}$ & 58 \\
& & & $8 \mathrm{mM}$ & 16 \\
\hline
\end{tabular}

${ }^{\#}$ Average cell counts from triplicate wells $(n=3)$; the coefficient of variation was $<10 \%$. 
Table 2. Comparison of chemosensitivity $\left(\mathrm{IC}_{50}\right)$ of ES-GFP and HT-29-GFP cells to 5-FU, gemcitabine, and sodium butyrate under different culturing conditions.

\begin{tabular}{lcccccc}
\hline & \multicolumn{3}{c}{ ES cells } & \multicolumn{3}{c}{ Colon cancer calls } \\
\cline { 2 - 7 } & $\begin{array}{l}5-\mathrm{FU} \\
(\mu \mathrm{M})\end{array}$ & $\begin{array}{c}\text { Gemcitabine } \\
(\mu \mathrm{M})\end{array}$ & $\begin{array}{c}\text { Sodium } \\
\text { butyrate } \\
(\mathrm{mM})\end{array}$ & $\begin{array}{c}5-\mathrm{FU} \\
(\mu \mathrm{M})\end{array}$ & $\begin{array}{c}\text { Gemcitabine } \\
(\mu \mathrm{M})\end{array}$ & $\begin{array}{c}\text { Sodium } \\
\text { butyrate } \\
(\mathrm{mM})\end{array}$ \\
\hline 2-D & 0.20 & 0.001 & 1.0 & 1.6 & 0.0043 & 1.2 \\
3-D low-density & 0.27 & 0.006 & 0.64 & 140 & 0.0036 & 6.0 \\
3-D high-density & $>770^{\mathrm{a}}$ & $>100^{\mathrm{a}}$ & 1.0 & $77-770^{\mathrm{b}}$ & $100-1000^{\mathrm{c}}$ & $\sim 36$ \\
\hline Resistance factor (Rf) & $>3850$ & $>10^{5}$ & 1 & $48-480$ & $>2.3 \times 10^{4}$ & 30 \\
\hline
\end{tabular}

Based on triplicate wells $(n=3)$; the coefficient of variation was $<10 \%$.

$\mathrm{IC}_{50}$ was significantly higher $(p<0.05)$ for 3 -D low-density cultures compared to 2-D cultures except for 5-FU for ES cells and gemcitabine for colon cancer cells. Except for sodium butyrate for ES cells, 3-D high-density cultures showed dramatically increased $\mathrm{IC}_{50}$ values compared to 3-D low-density and 2-D cultures.

${ }^{a}$ No significant cytotoxicity at the highest dose studied

${ }^{\mathrm{b}}$ Caused significant growth inhibition at $77 \mu \mathrm{M}$ and early cell death at $770 \mu \mathrm{M}$

${ }^{\mathrm{c} C a u s e d ~ s i g n i f i c a n t ~ g r o w t h ~ i n h i b i t i o n ~ b u t ~ n o ~ a p p a r e n t ~ c e l l ~ d e a t h . ~}$

$\mathrm{Rf}=\mathrm{IC}_{50,3-\mathrm{D} \text { high-density }} / \mathrm{IC}_{50,2-\mathrm{D}}$ 


\section{Figure Caption}

Fig. 1. Microbioreactors on modified 384-well plate used in the 3-D cytotoxicity assay. Each microbioreactor was made of 9 original wells on the plate with their walls removed. A PET matrix as cell scaffold was placed in the original center well for the 3-D culture.

Fig. 2. ES and HT-29 cells cultured in nonwoven fibrous PET matrices. A, B: ES-GFP and HT29-GFP under fluorescent microscope showing cells emitting green fluorescent light. C, D: SEM photos of cells showing 3-D cell aggregates similar to in vivo mature tissues or tumors.

Fig. 3. Growth kinetics of 3-D cultures of ES-GFP (A-C) and HT-29-GFP (D-F) exposed to 5FU (top), gemcitabine (middle), and sodium butyrate (bottom) in a dose-dependent manner. Drugs were added at $24 \mathrm{~h}$ or one day after inoculation.

Fig. 4. Drug response kinetics of 3-D high-density cultures of ES-GFP (A-C) and HT-29-GFP (D-F) exposed to 5-FU (top), gemcitabine (middle), and sodium butyrate (bottom) in a dosedependent manner. Drugs were added after cells had developed large aggregates after culturing for several days (indicated by arrow). Fluorescence signals were normalized with respect to the intensity at the time of drug addition for easier comparison.

Fig. 5. Images of fresh human tonsil tissue (left), HT-29 cells in 3-D high-density cultures (middle) and agarose-embedded cell pellets from 2-D monolayer culture (right) immunohistochemically stained with quiescence marker p27kip1 (top row) and proliferation marker Ki-67 (bottom row), respectively. 


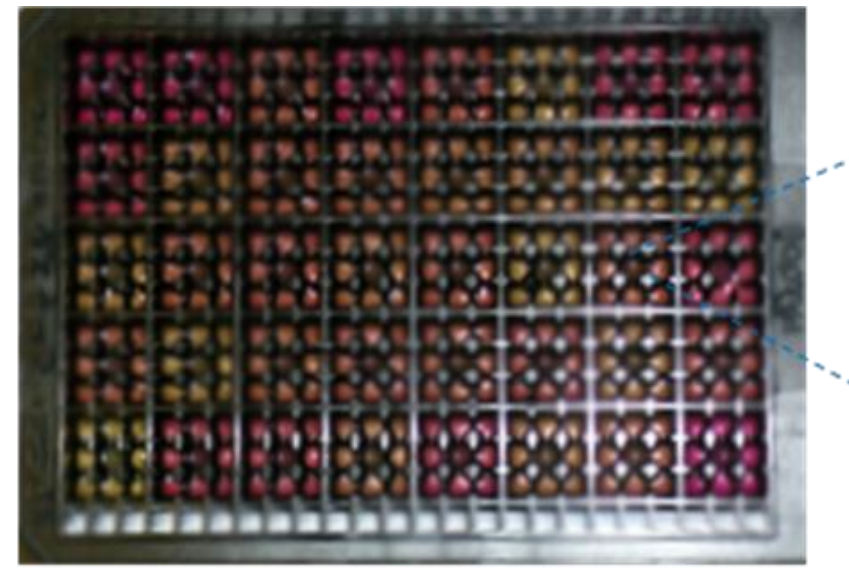

Modified 384-well plate

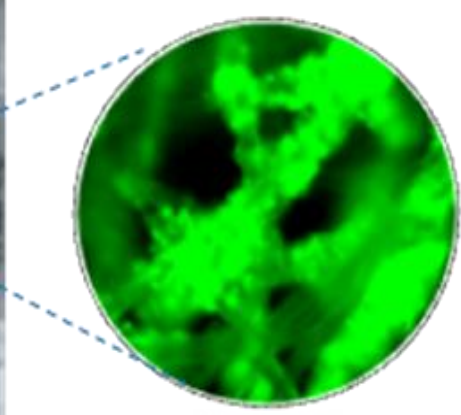

\section{GFP-cells in 3-D} PET scaffold

Fig. 1. Microbioreactors on modified 384-well plate used in the 3-D cytotoxicity assay. Each microbioreactor was made of 9 original wells on the plate with their walls removed. A PET matrix as cell scaffold was placed in the original center well for the 3-D culture. 

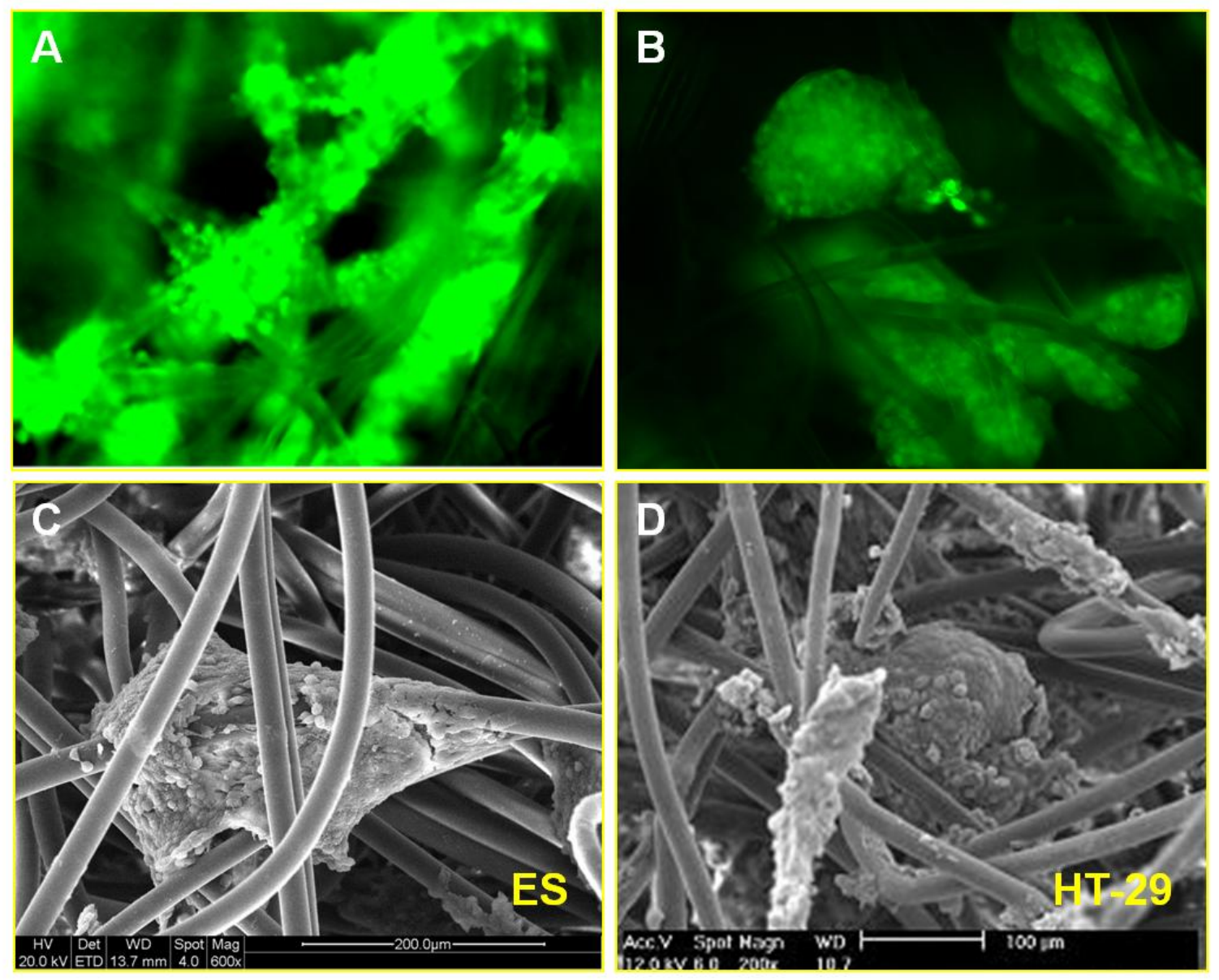

Fig. 2. ES and HT-29 cells cultured in nonwoven fibrous PET matrices. A, B: ES-GFP and HT29-GFP under fluorescent microscope showing cells emitting green fluorescent light. C, D: SEM photos of cells showing 3-D cell aggregates similar to in vivo mature tissues or tumors. 

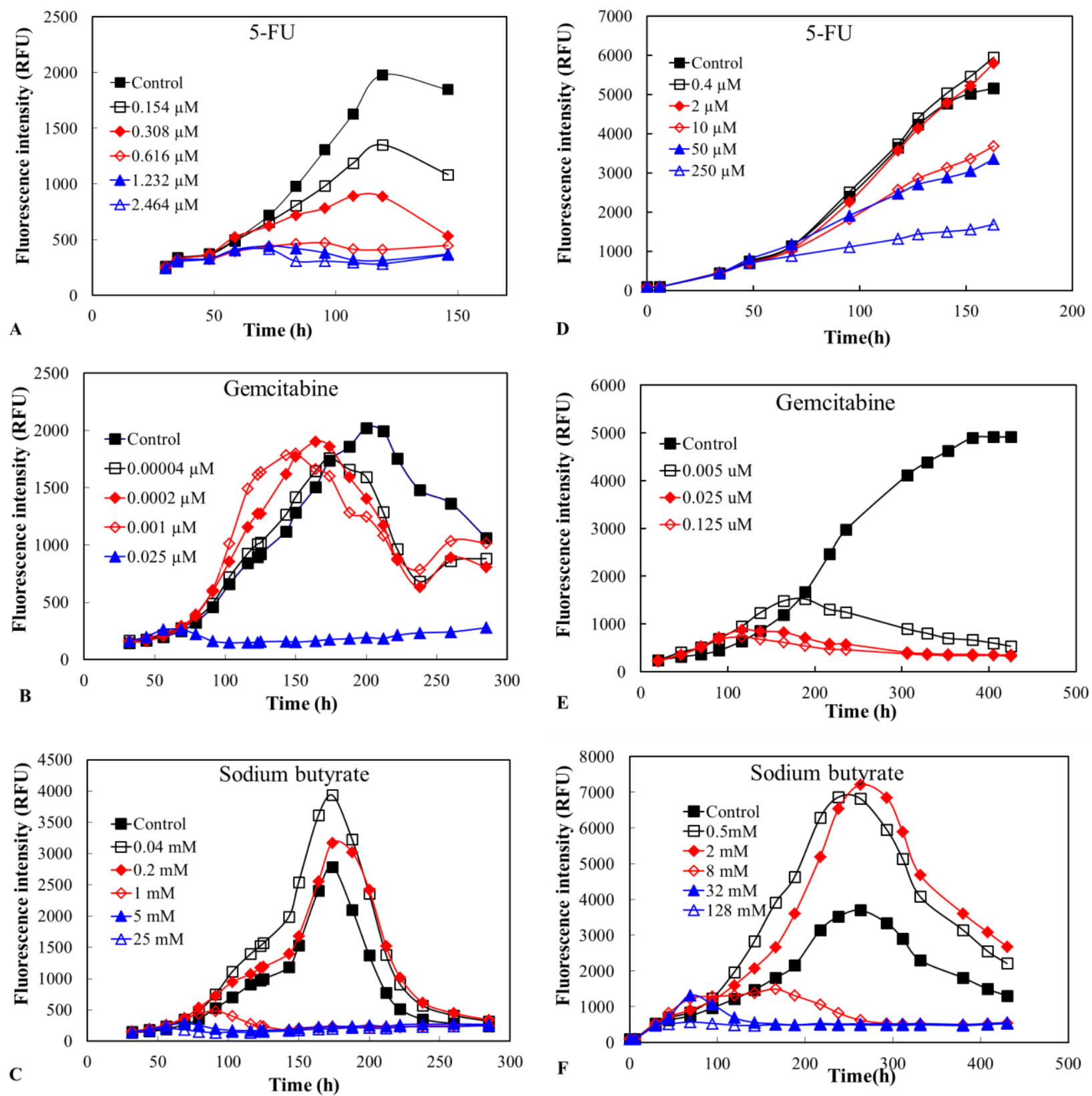

Fig. 3. Growth kinetics of 3-D cultures of ES-GFP (A-C) and HT-29-GFP (D-F) exposed to 5FU (top), gemcitabine (middle), and sodium butyrate (bottom) in a dose-dependent manner. Drugs were added at $24 \mathrm{~h}$ or one day after inoculation. 

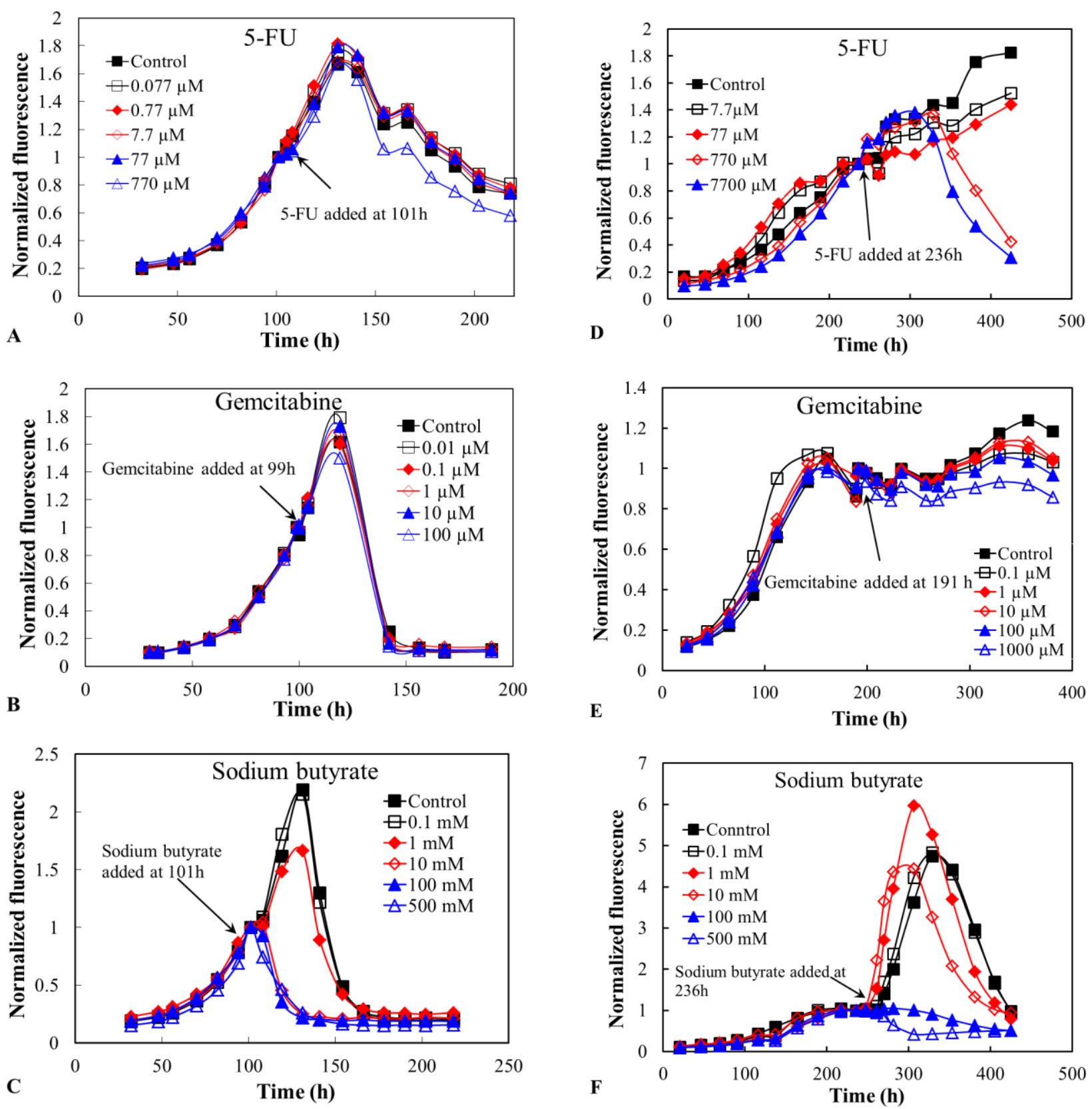

Fig. 4. Drug response kinetics of 3-D high-density cultures of ES-GFP (A-C) and HT-29-GFP (D-F) exposed to 5-FU (top), gemcitabine (middle), and sodium butyrate (bottom) in a dosedependent manner. Drugs were added after cells had developed large aggregates after culturing for several days (indicated by arrow). Fluorescence signals were normalized with respect to the intensity at the time of drug addition for easier comparison. 

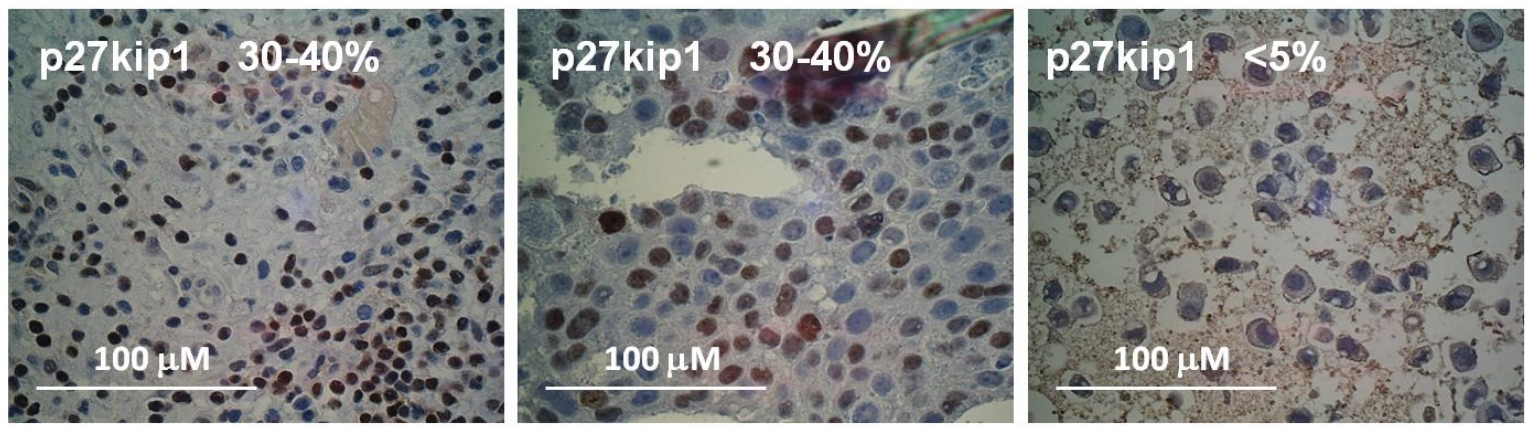

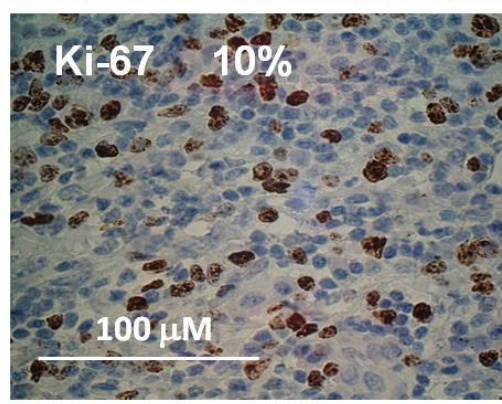

Fresh tonsil tissue

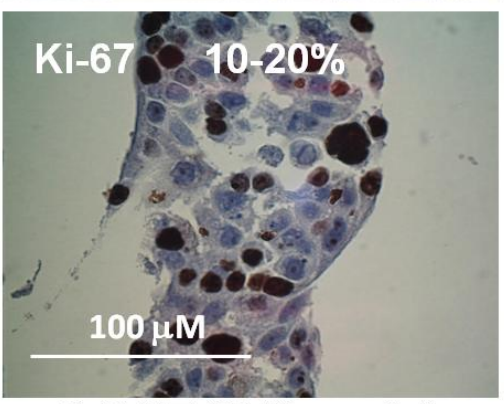

3-D HT-29 (2 week )

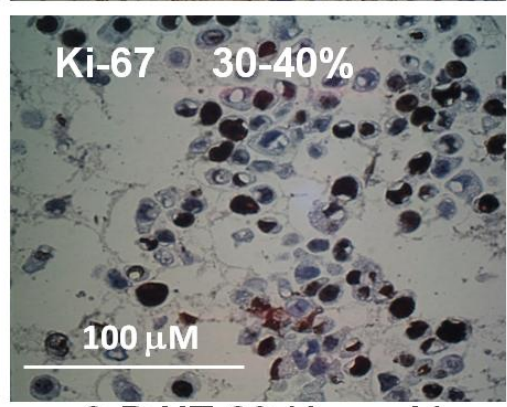

2-D HT-29 (1 week)

Fig. 5. Images of fresh human tonsil tissue (left), HT-29 cells in 3-D high-density cultures (middle) and agarose-embedded cell pellets from 2-D monolayer cultures (right) immunohistochemically stained with quiescence marker p27kip1 (top row) and proliferation marker Ki-67 (bottom row), respectively. 


\section{Graphical Abstract}

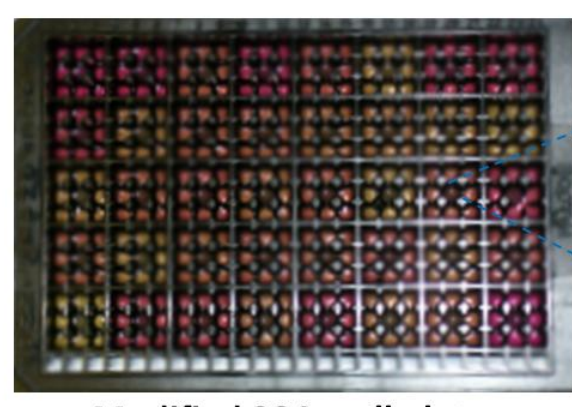

Modified 384-well plate PET scaffold
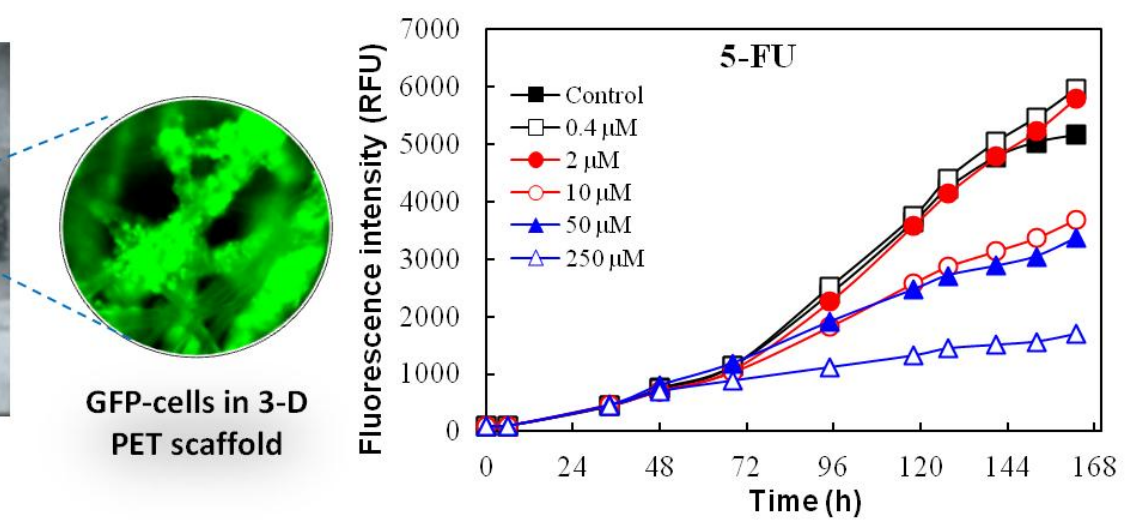

Microbioreactors on modified 384-well plate with 3-D PET scaffold for growing cells expressing green fluorescent protein (GFP) in response to drug as a multicellular model for high throughput cytotoxicity and drug efficacy screening 